\title{
Interappointment Flare-up in Endodontics: A Case Report and an Overview
}

Vanitha Shenoy, Rahul Kumar, MV Sumanthini

\begin{abstract}
A problem that an endodontist can encounter during the course of root canal treatment is pain and swelling or both in the form of flare-ups. The causes for this flare-up are numerous which include mechanical, chemical, microbial injury to the pulp or periradicular tissues, whereby an acute periradicular inflammation results. This situation can be prevented by selection of instrumentation technique, that extrude lesser amount of debris apically, completion of biomechanical preparation in a single sitting, use of antimicrobial intracanal medicament between appointment in the treatment of infected cases with an appropriate coronal temporary restoration and maintaining asepsis throughout the root canal treatment. The flare-up phenomena are complex and not well-understood which involves a number of hypotheses for its etiology. A correct diagnosis and treatment aids in the resolution of the flare-up. This case report explains the management of an interappointment flare-up, with an overview of flare-ups.
\end{abstract}

Keywords: Root canal treatment, Pain, Swelling, Flare-up.

How to cite this article: Shenoy V, Kumar R, Sumanthini MV. Interappointment F lare-up in Endodontics: A Case R eport and an Overview. J Contemp Dent 2012;2(2):53-56.

\section{Source of support $\mathrm{Nil}$}

Conflict of interest: None declared

\section{INTRODUCTION}

The interappointment flare-up is a true complication where, within a few hours to a few days, after an endodontic procedure a patient has significant increase in pain or swelling or a combination of the two, wherein the patient must come in for an unscheduled visit, for emergency treatment. ${ }^{1,2}$ The incidence of flare-ups is low and occurs only in a small percentage. M orse et al reported an incidence of approximately $20 \%$ flare-ups where swelling was the only criteria, after treating asymptomatic teeth with pulp necrosis and chronic apical periodontitis. ${ }^{3}$ In contrast, Barnett and Tronstad in a retrospective study determined an incidence of approximately $5.5 \%$ flare-ups, where pain and/or swelling were the criteria, in patients with a similar diagnosis of pulp necrosis with asymptomatic periapical lesion, but $1.4 \%$ in all patients regardless of diagnosis. ${ }^{4}$

Thus, the incidence of flare-ups increases in direct relationship to the severity of the patient's preoperative pathosis and signs/symptoms. The lowest frequency of occurrence is generally with a vital pulp without periapical pathosis, the highest frequency with patients who present with more severe pain and swelling, with pulp necrosis and acute apical abscess. ${ }^{2}$ Seltzer $S$ and Naidorf ${ }^{5}$ explained possible etiological factors for flare-ups and stated the reasons for such exacerbations as not always clear and explained some hypothesis which are interrelated. Different approaches and techniques have been mentioned for the management of flare-ups. ${ }^{6}$

\section{CASE REPORT}

A 20-year-male patient presented to the Department of Conservative Dentistry and Endodontics, M GM Dental College and Hospital, Navi M umbai, with severe pain and a large swelling on the right side of the face, extending from the lower border of the mandible involving the lower eye lid (Figs $1 A$ and $B$ ), since 3 days. The patient gave a history of discontinued root canal treatment in relation to the maxillary right first molar (26). The intraoral examination revealed an access cavity that had been prepared devoid of temporary restoration. The patient had an intraoral periapical radiograph from his previous discontinued root canal treatment, which showed radiolucency in the crown and at the apex of the mesial $\operatorname{root}$ (Fig. 1C). The concluding diagnosis for 26 was an acute apical abscess (flare-up). The patient's medical history was noncontributory. The patient was explained of the problem and reassured.

For the flare-up emergency appointment, under rubber dam isolation (Hygienic Dental Dam, Coltene/W haledent Inc), (Fig. 1D), the access cavity and the root canal was irrigated with normal saline. The access cavity was modified, root canals located. Four root canals were located, mesio-buccal, distobuccal, palatal and mesiobuccal 2 (M B 2) (Fig. 2A). W orking length was determined using an apex locator (Propex, Dentsply) and confirmed with an intraoral periapical radiograph (see Fig. 2A). Biomechanical preparation was completed using ProTaper $\mathrm{Ni}-\mathrm{Ti}$ rotary instrument (D entsply, M aillefer) with a crown-down technique, under copious irrigation with normal saline, followed by $5 \%$ sodium hypochlorite solution (D entpro, Chandigarh, India). Calcium hydroxide saline paste was placed in the root canal and a closed dressing of zinc oxide eugenol (DPI, M umbai, India) of a thin consistency was placed in the access cavity. The patient was prescribed antibiotics and analgesics for 5 days. 
The patient was contacted daily until the signs and symptoms subsided (Fig. 2B). A fter 7 days, the swelling had subsided and the patient was asymptomatic. Obturation of the root canal was completed using cold lateral compaction of gutta-percha (Dentsply, M aillefer) using $\mathrm{AH}$ Plus sealer (D entsply, $M$ aillefer; Figs $2 C$ and $D$ ). The access cavity was subsequently restored with silver amalgam (Dispersalloy, Dentsply, M aillefer). The patient was advised to have a full coverage crown with 26.
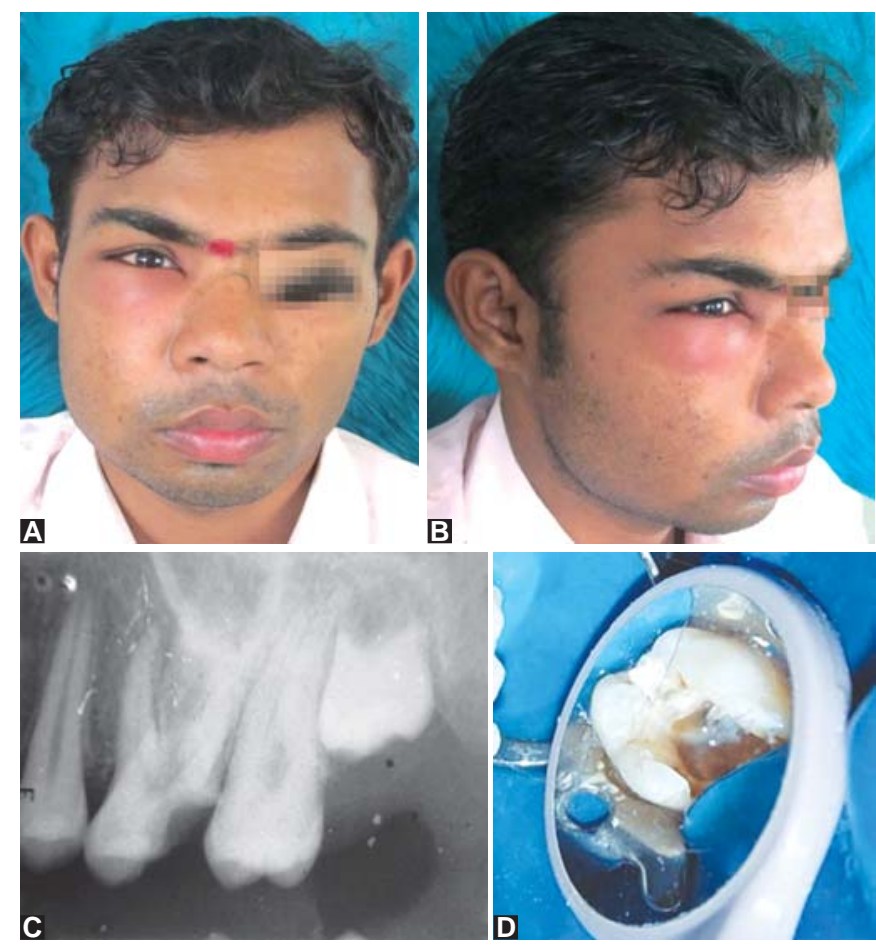

Figs 1A to D: Extraoral swelling, (A) frontal view, (B) lateral view, (C) intraoral preoperative radiograph, (D) clinical photograph of access opening

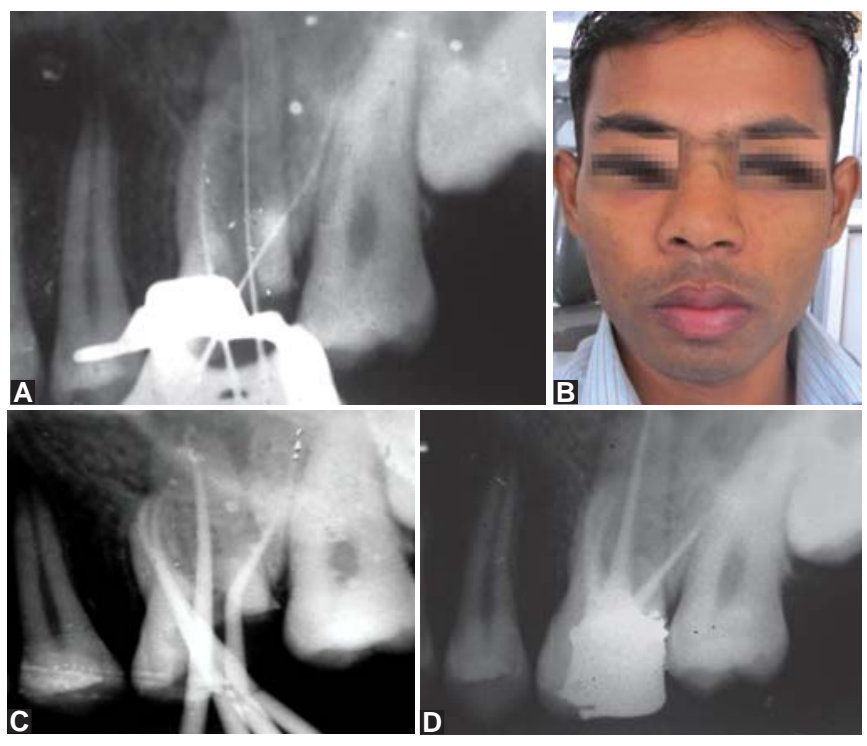

Figs 2A to D: (A) Working length radiograph, (B) 7th day postoperative photograph shows extraoral swelling has subsided, (C) master cone radiograph, (D) postoperative radiograph

\section{DISCUSSION}

Pain and swelling are the most common sign and symptom that can occur during flare-up. The causes for this could be an 'al teration of the local adaptation syndrome' where Selye demonstrated this factor by injecting air subcutaneously into the backs of rats, causing the air-filled tissues to balloon out. He then injected various chemicals into this air-filled pouch, creating an acute inflammatory response, in the form of a 'granuloma pouch', wherein the pouch was lined with granulation tissue. Subsequently the pouch was injected with the same chemical irritants which had produced the original inflammation and it was observed that there was no reaction and the tissues had adapted to the irritant. Evacuation of the content of the pouch resulted in healing but when a new and different irritant was injected into the pouch, a violent reaction leading to tissue necrosis occurred. In a clinical situation, inflammation of periapical lesion may be adapted to the irritant and chronic inflammation may exist without perceptible pain or swelling. However, when endodontic therapy is performed, a new irritant in the form of medicaments, irrigating solutions or tissue proteins, altered by chemicals may be introduced into the granulomatous lesion and then a violent reaction may follow, leading to liquefaction necrosis, indicating of an alteration, thus showing that there is a local tissue adaptation to applied irritants. $^{7}$

A change in periapical tissue pressure is another cause wherein measurements of periapical tissue pressure during endodontic therapy in dogs teeth, revealed that both the negative and positive pressures occur. ${ }^{8}$ The pressure fluctuated over an 8 hours period. In teeth with increased periapical pressure excessive exudates, not removed by the lymphatics, would tend to create pain by pressure on nerve endings. When the root canals of such teeth were opened the fluid would tend to be forced out. In contrast should the periapical pressure be less than atmospheric pressure, microorganisms and al tered tissue proteins could be aspirated into the periapical area resulting in accentuation of the inflammatory response and severe pain. ${ }^{5}$

$\mathrm{M}$ icrobes are one of the important causes of flare-ups. Prior to 1970's voluminous studies of the flora of infected root canals showed the presence of a considerable variety of microorganisms. Based on recent studies anaerobic culturing techniques produce a far greater spectrum of microbial isolates than purely aerobic techniques. ${ }^{9}$ A naerobes in mixed root canal infections may be responsible for the production of enzymes and endotoxins, the inhibition of chemotaxis and phagocytosis, and endotoxins, and interference with antibiotic activity resulting in the persistence of painful periapical lesions. ${ }^{10}$ Bacteriodes 
melaninogenicus, an anaerobic, Gram-negative rod, is present in combination with other microorganism, which produce endotoxin, which activates the Hageman factor, which leads to the production of bradykinin, a potent pain mediator. The endotoxins are capable of resisting ingestion by polymorphonuclear leukocytes, even after ingestion, intracellular killing is impaired ${ }^{11}$ and in the presence of complement, endotoxin also enhances inflammation through the release of vasoactive chemicals. ${ }^{12} \mathrm{Gram}$-positive bacteria are also involved in root canal flare-ups. Whether the flora of an infected root canal can change when endodontic treatment is performed or whether a change in the proportional of aerobes to anaerobes can cause clinical exacerbations are still conjectural. . $^{2,13}$

The effect of chemical mediators during inflammatory response can be derived from cells or plasma, which includes histamine, serotonin, prostaglandins, platelet-activating factor, leukotrienes, various lysosomal components and some lymphocyte products called lymphokines, all of which are capable of causing pain. The plasma mediators are present in the circulation, the Hagmen's factor (factor XII), which when activated, are the cause for pain. Neutrophil products when the root canal is instrumented, an acute inflammatory response is initiated in the periapical tissues. $\checkmark$ arious chemical mediators are released endogenously or by inflammatory cells in acute periodontitis, which cause pain. ${ }^{5,13} \mathrm{~A}$ change in cyclic nucleotide that is the cyclic adenosine mono phosphate (AM P) is the second messenger for many hormones, transmitting information to the interior of the cell. Transmitters, such as histamine or epinephrine and serotonin, elaborated during the inflammatory response, are capable of el evating cyclic A M P levels in the periapical tissues. The cyclic guanosine monophosphate (GM P) is al so a second cyclic nucleotide, which is present in all living systems. Cellular regulations, including pain transmission, may be influenced by the interaction of cyclic AMP and cyclic GM P. 5,13

Immunological factors also play a role in chronic pul pitis and periapical periodontitis, the presence of macrophages and lymphocytes indicates that both cell-mediated and humoral immune reactions are involved. Despite their protective effects immunological mechanisms may contribute to the destructive phase of inflammation. A ntigens from medicament-al tered tissue, antigen-antibody complexes, and root canal filling materials have been reported to be capable of invoking immunological reactions. ${ }^{14}$ Pulp and periapical destruction may then be the result of a shift in the production of IgG over IgA, causing perpetuation and aggravation of the inflammatory process. ${ }^{15}$ Other possibilities for flare-ups may be based on activation of the kallikrein-kinin and coagulation systems, by the binding of IgG or IgM to cell surface antigens, and by the subsequent involvement of the complement system. ${ }^{5,13}$ Finally, the various psychological factors, such as fear of dentists and dental procedures, anxiety, apprehension, and many other psychological factors influence the patient's pain perception and reaction thresholds ${ }^{16,17}$ to the initiation of flare-ups.

As the etiological factors often cannot be precisely determined, many treatment options have been suggested. ${ }^{6}$ Cohen advocated occlusal relief prior to endodontics ${ }^{18}$ for the prevention of postoperative pain but other endodontists recommended occlusal relief prior to endodontic therapy only in teeth with painful periapical symptoms ${ }^{19}$ whereas some reduce the occlusion of teeth undergoing endodontic therapy when painful symptoms develop. ${ }^{20}$

Establishment of drainage is the most effective method for reducing pain and swelling. This can be accomplished by removing the temporary dressing from the access cavity. In most cases the accumulated exudates will drain down the root canal affording immediate relief. If no exudate emerges, the root canal may be blocked by packed dentinal shavings in the apical third of the root canal. Passing a sterile root canal file or reamer through this material hel ps establish the flow of exudates. In some the exudates may be absent or cannot be evacuated through the root canal, surgical intervention is then the choice of treatment. It is advised to temporarily close the access cavity ${ }^{18,21}$ rather than leave it open for drainage, as the salivary products increase bacterial growth, introduces new microorganisms that activates the al ternate complement pathway and may enhance bradykinin, leading to production of pain.

Intracanal medicaments are used to afford relief from painful exacerbations during root canal therapy. Among them are antimicrobial agents, such as formocresol, cresatin, eugenol, camphorated monochlorphenol and iodinepotassium iodide have been studied and there has been no significant relationship between flare-up and type of therapy used. Irrigating solution choice makes little difference in the incidence of postoperative discomfort, as long as the irrigating solution does not get forced beyond the foramen of the tooth. It is also difficult to attribute lower pain incidence specifically to the use of any particular irrigant. Sulfa compounds and corticosteroids also have been used in the management flare-ups but the results were not promising. ${ }^{6}$ Systemic drugs in the form of antibiotics have been locally and systemically used for the relief of pain against various strains of organisms during endodontic therapy. There is no specific antibiotic that is capable of reducing or eliminating painful exacerbations during endodontic therapy. ${ }^{6} \mathrm{~N}$ onnarcotic analgesics relieve pain without al tering consciousness, which are effective against 
most pain of dental origin. N onsteriodal anti-inflammatory agents are drug of choice for mild to moderate pain. N arcotic anal gesics are commonly prescribed for relief of severe pain. However, the prescription of the analgesic has to be in accordance with the signs and symptoms. ${ }^{6}$

The follow-up care of a patient with a flare-up should be by contacting the patient daily until the symptoms subside. Communication may be made by telephone or by the patient reporting to the clinic. For those patients with severe or persistent problems which do not resolve, then additional treatment procedures are required. ${ }^{1}$

\section{CONCLUSION}

Signs and symptoms of flare-ups may be severe in magnitude, they are rarely serious, i.e. not life-threatening. They are localized and do not involve structures other than those oral or local perioral. The condition and the cause have to be diagnosed, followed by a proper treatment plan, to treat the flare-up successfully.

\section{REFERENCES}

1. W al ton RE. Interappointment flare-ups: Incidence, related factors, prevention, and management. Endo Topics 2002;3: 67-76.

2. Siqueira J F J r. M icrobial causes of endodontic flare-ups-review. Int Endod J 2003;38:453-63.

3. Morse Dr, Koren LZ, Eposito JV, et al. Infections flare-ups: Induction and prevention. Int J Psychosom 1983;33:5-63.

4. B arnett $F$, Tronstad $L$. The incidence of flare-ups following endodontic treatment. J Dent Res 1989;68:1253.

5. Seltzer S, Naidorf IJ. Flare-ups in endodontics: I. Etiological factors. J Endod 2004;30:476-81.

6. Seltzer S, N aidorf IJ . Flare-ups in endodontics: II. Therapeutic measures. J Endod 2004;30:482-90.

7. Selye $H$. The part of inflammation in the local adaptation syndrome. In: Jasmin G, Robert A (Eds). The mechanism of inflammation. Acta M ontreal 1953;53-74.

8. Herzig E, R oot WS. R elation of sympathetic nervous system to blood pressure of bone marrow. Am J Physiol 1959;196:105.

9. Zielke Dr, Heggers JP, Harrison JW. A statistical analysis of anaerobic versus aerobic culturing in endodontic therapy. Oral Surg 1976;42:830.
10. Dahle'n G, Bergenholtz G. Endotoxic activity in teeth with necrotic pulps. J Dent Res 1980;59:1033.

11. Sundqvust GK, Eickerborn MI, Larsson AP, Sjogren UT. Capacity of anaerobic bacteria from necrotic dental pulps to induce purulent infections. Infect Immune 1979;25:685.

12. Mergenhagen SE. Complement as a mediator of the inflammatory response: Interaction of complement with mammalian and bacterial enzymes. J Dent Res 1972;51:251.

13. Seltzer S. Pain in endodontics. J Endod 2004;30:501-03.

14. Block RM , L ewis RD, Sheats J B, B urkeSh, Fawley J. A ntibody formation and cell-mediated immunity to dog pulp tissue al tered by eight endodontic seal ers via the root canal. IntEndod J 1982; 15:105.

15. B randtzaeg P. Immunology of inflammatory periodontal lesions. Int Dent J 1973;22:438.

16. ScottDS, Hirschman R. Psychological aspects of dental anxiety in adults. J A m Dent A ssoc 1982;104:27.

17. Berggren U, M eynert G. Dental fear and avoidance: Causes, symptoms and consequences. J A m Dent A ssoc 1984;109:247.

18. Cohen S. Endodontic emergiencies. In: Cohen S, Burns RC, (Eds). Pathways of the pulp (2nd ed). St L ouis: CV M osby Co, 1980;31.

19. Grossman LI. Endodontic practice (10th ed). Philadelphia L ea and Febiger, 1981;85:93.

20. Creech J L, W al ton $R E, K$ al tenbach $R$. Effect of occlusal relief on endodontic pain. J A m D ent A ssoc 1984;109:64.

21. A ugust DL. M anaging the abscessed open tooth: I nstrument and close-part 2. J Endod 1982;8:364.

\section{ABOUT THE AUTHORS}

\section{Vanitha Shenoy (Corresponding Author)}

Professor and Head, Department of Conservative Dentistry and Endodontics, MGM Dental College and Hospital Navi M umbai, M aharashtra, India, Phone: +919167629730 e-mail: vanithashenoy@gmail.com

\section{Rahul Kumar}

Senior Lecturer, Department of Conservative Dentistry and Endodontics, MGM Dental College and Hospital, Navi Mumbai M aharashtra, India

\section{Sumanthini}

Professor, Department of Conservative Dentistry and Endodontics M G M Dental College and Hospital, Navi M umbai, M aharashtra, India 